\title{
Ultra-fast Lithium cell charging architecture for Mission Critical Applications
}

\author{
${ }^{1}$ Arif Sari, ${ }^{2}$ Behnam Rahnama and ${ }^{3}$ Ersin Caglar, \\ ${ }^{1}$ School of Applied Sciences, Department of Management Information, \\ European University of Lefke, Cyprus \\ ${ }^{2}$ Department of Computer Engineering, Okan University, Istanbul, Turkey \\ ${ }^{3}$ Department of Computer Engineering, European University of Lefke, Cyprus; \\ asari@ieee.org ; rahnama@ieee.org ; erscaglar@gmail.com
}

\begin{abstract}
This research presents design and implementation of the ultra-fast parallel lithium charging architecture with an embedded algorithm using an active PWM charge pump supported by a hybrid control mechanism consisting of Temperature, humidity and current sensors. The new architecture guaranties the ultra-fast parallel charging cycles of lithium cells without lifespan reduction due to possible overheat side effects in mission critical applications.
\end{abstract}

Keywords: Lithium Cell, Fast Charging, PWM Charge Pump, Mission Critical Application.

\section{Introduction}

Lithium batteries are used everywhere in our daily life from cellular phones to handheld devices, notebooks and tablet PCs. Chargers basically protect cells by limiting the charge cycle from over-current and over-voltage. Such chargers normally are used in living environmental temperature and humidity. On the other hand, mission critical applications require dedicated and specifically designed architectures to overcome challenging temperature and humidity levels.

In such situation, charging circuitry and cells are kept in a temperature controlled area while exterior climate change would not affect charging performance. However, providing such a safe protective cover is not always feasible everywhere. Wireless Sensor Network nodes might be placed in any climate and the usual expectation is their durability. However, the limited space does not allow implementation of a perfect protection case to keep the cells temperature in a steady state. Use of ultra-fast charge pump circuitry is vital in satellites and spacecraft missions while the temperature exceeds our living limits toward extremities.

In addition, charging circuitry must be fault tolerant and self-recoverable after any power failure. This can be managed by applying watchdog timers and various timing stages in a cascade control to make sure the reliability of the system.

The core implementation is to capture the voltage level and passing current at each cell, battery temperature and surrounding humidity. These raw data are processed and a fast phase and frequency correct PWM generator compare match value is updated. 
Using hardware timer allows the microcontroller to jump to different power saving stages and utilizes less energy while controlling the charging circuitry.

The PWM frequency for switching the parallel charging circuit per channel is fixed on $1 \mathrm{KHz}$. The duty cycle varies from $0 \%$ for total de-charge cycle to $100 \%$ for total charging cycle according to the aforesaid measuring variables.

Next section covers a survey on various lithium charge management integrated circuits and similar challenges in the literature. Then we provide our proposed novel and simple to implement but yet efficient charging algorithm and circuit for mission critical applications. Finally, this research paper is concluded by findings and detailed prospective study.

\section{Related Work}

Due to growing interest in flexible energy storage devices, scientists in South Korea proposed a method to tackle with charging duration of lithium ion rechargeable batteries [1]. The proposed approach is related with the use of cathode material, standard lithium manganese oxide (LMO), soaked in a solution containing graphite. Recharging process of the battery proposed through a network of conductive traces which runs throughout the cathode graphite-soaked LMO. A lithium ion battery consists of several parts where the carbonized graphite networks recharge all parts of the battery simultaneously. Researchers' speed-up the recharging process and decreased the amount of time to recharge lithium ion batteries through a specific method. An electrolyte and graphite anode is used to package the cathode and all energy holding particles of the battery start recharging simultaneously by this way which leads to speed up of charging lithium ion batteries. In the literature, another group of researchers demonstrated a lightweight, thin and flexible full lithium ion battery with high-rate performance and energy density [2]. The proposed battery model was loaded with $\mathrm{Li}_{4} \mathrm{Ti}_{5} \mathrm{O}_{12}$ (Lithium Titanate - LTO) and $\mathrm{LiFePO}_{4}$ (Lithium Iron Phosphate - LFE). The Lithium Titanate is a prospective material for anodes of Li-ion batteries. The LTO leads Li-ion batteries to expand into different applications such as electrical cars and hybrid vehicles. The usage of LTO in Li-ion batteries dramatically increases the battery lifetime and provides wide range of operation possibilities under different operation temperatures such as $-30^{\circ} \mathrm{C}-60^{\circ} \mathrm{C}[3]$.

The charging of Li-ion batteries requires specific attention in order to prevent overcharging and thermal runway problems where these problems may lead to damage in Li-ion batteries. Extended charging of Liion batteries may lead to a change in forms of battery when it is above $4.30 \mathrm{~V}$. This change can be observed on the batteries as changes in plating of metallic lithium on the anodes, while the cathode material becomes an oxidizing agent, loses stability and produces carbon dioxide $\left(\mathrm{CO}_{2}\right)$ [4]. As it is mentioned before, Li-ion batteries consist of cells and cell pressure can rise due to overcharging. The usage of LTO in Li-ion batteries decreases the amount of charging while preventing battery against damage due to overcharging.

LFE, is a similar component in terms of their usage in high power applications likewise Hybrid vehicles. The usage of LFE cells in Li-ion batteries leads non-explosive and long battery life cycle for them however their energy density is lower than normal Li-Ion cells. These anode materials, LFE and LTO, are used to improve performance and durability of the Li-ion batteries by the researchers. The LTO is introduced for sodium ion-batteries to extend lifetime and provide fast charging facilities [4]. 
Arif Sari, Behnam Rahnama \& Ersin Caglar; Ultra-fast Lithium cell charging architecture for mission critical applications, Transactions on Machine Learning and Artificial Intelligence, Volume 2 No 5 Oct (2014); pp: 10-16

In order to meet the modern society needs for flexible and durable energy storage devices, another research has conducted to propose a new structure of thin and flexible Li-ion battery design [5]. The goal of proposed design was to reach to high energy density with mechanical flexibility. The Li-ion battery materials are coated with thin carbon nanotube (CNT) films. The flexibility is provided through CNT design where it acts as a current collector for both anode and cathode. Since the actual performance of rechargeable Li-ion batteries depends on the thermodynamics and kinetics of the electrochemical reactions involved in the components of the cells such as reductant, oxidant, electrolyte and separators, the usage of nanotechniques and nanomaterials becomes an essence of designing rechargeable Li-ion batteries. The usage of different nanotechniques and nanomaterials such as $\mathrm{LiCoO} 2, \mathrm{LiFePO}_{4}, \mathrm{LiMn}_{2} \mathrm{O}_{4}$, with $\mathrm{Li}_{4} \mathrm{Ti}_{5} \mathrm{O}_{12}$ and $\mathrm{LiMn}_{2} \mathrm{O}_{4}$ aims to increase lifetime, durability and energy density of rechargeable Li-ion batteries without affecting safety and reliability issues. Variety of researches conducted on implementation of different nanomaterials on development of rechargeable Li-ion batteries where the researches are spread to hybrid electric vehicles, clean energy and aerospace fields [6].

Researchers focused on rechargeable Li-ion batteries toward the electron transfer on key materials and components of Li-ion batteries on molecular, atomic or ionic transport for to solve long cycle, extended energy diversification and reliability issues [7]. Variety of functional materials which are used to design rechargeable Li-ion cells such as lead acid, nickel-metal hydride and sodium-sulfur batteries are reviewed. However, implementation of fast charging mechanisms or architectures are not proposed or examined to expose the impact of nanomaterials on cell design and safety, reliability and lifetime issues of rechargeable Li-ion batteries.

\section{Proposed Ultra-Fast Charging Architecture}

\subsection{Materials}

Lithium cells are sensitive to over-voltage and under-voltage cycles. Thresholds for minimum and maximum voltage level as well as charging cycle start and end latency may be controlled by local charging integrated circuit. A widely used example of such chips is S-8254A. Several lithium charge management ICs also support a PTC or an NTC resistor on cell to cutoff charging in case the cell temperature exceeds a certain threshold. MCP73844 series of Microchip battery management chips can be considered as a wellknown IC in this category. However, all aforementioned ICs are designed for living environmental temperature range.

Humidity might not play an important role as usually charging circuitry can be isolated in a closed plastic like packaging such as laptop chargers. The Environmental humidity does not have considerable effect on charger. However, temperature is the main factor we must take into account. Maxim DS18S20 HighPrecision 1-Wire Digital Thermometer was used in test platform to catch temperature changes from $-55^{\circ} \mathrm{C}$ to $+125^{\circ} \mathrm{C}$ degrees.

This is known that the extreme temperature levels on earth are $-88^{\circ} \mathrm{C}$ at Vostok Station in Antarctica to $+58^{\circ} \mathrm{C}$ in the Libyan Desert. Of course, the entire design must be changed in case a WSN node powered by a lithium based solar charger is used at minimum temperature proximity.

Our designed platform so called "Adamus" consists of four blocks; first, Charging circuitry, second, Voltage, Current, temperature and humidity measurement sensors, third, microcontroller and Darlington 
switching gates and fourth, data logger over UART serial interface transferred over Serial to USB bridge (AS8303CDS). Other peripherals including LGM128128A LCD screen and buzzer help to better understanding of test case during runtime.

\subsection{Designed Hardware}

Parallel charging circuit is powered by FTD2017A on four separate channels on extension boards. As mentioned earlier, DS18S20 is used to catch the cell temperature. In addition, a combined temp/humidity sensor DHT11 sensor checks the charger circuit conditions. Voltage per cell is captured by ADC interface available at microcontroller. A semi electromagnetic transducer IC (ACS712ELC-05B) as separate board converts the current flow to voltage level and captured separately per each channel. Atmel xmega series of microcontrollers are more desired due to extreme low power consumption. However, this test board is managed by Atmega32, the older version but yet practical from laboratory purposes. ULN2803 are used to provide four coupled $n$-channel switching for all four parallel cells. Finally, captured data is transferred over the serial link to the host computer for logging purposes. A future amendment to the project is to include a microSD card onboard to save captured data without necessarily transferring them over serial link. Following figure presents the Adamus project PCB development board.

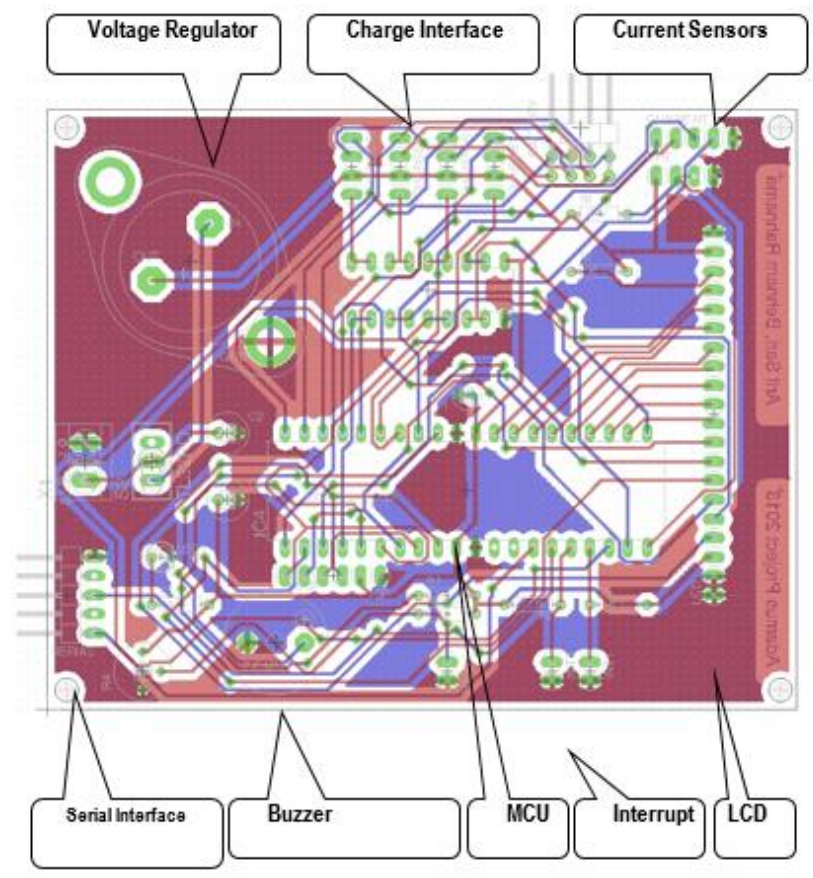

Figure.1 The charge Management Printed Circuit Board

\subsection{Charging Algorithm}

The charging algorithm basically considers three measurement variables namely, Cell Voltage (VCi), Charge Current (ICi), Cell temperature (TC). In addition, Board Temperature (TB) and Humidity (HB) are taken into account for board management purposes. As the result, compare value (PCi) in phase and frequency correct PWM mode is changed per each channel. The PWM duty cycle for each channel is defined as PDi. The modularity of design helps in parallelization of charging circuitry for more number of cells. 
Arif Sari, Behnam Rahnama \& Ersin Caglar; Ultra-fast Lithium cell charging architecture for mission critical applications, Transactions on Machine Learning and Artificial Intelligence, Volume 2 No 5 Oct (2014); pp: 10-16

The program captures aforementioned values and transfers them to the host at each second in addition to reset the watchdog timer value. Accordingly, read timeout on the host device is considered as two seconds. Watchdog timer is set to restart the program flow at each two second in case of system inactivity unless, total charging cycle is accomplished.

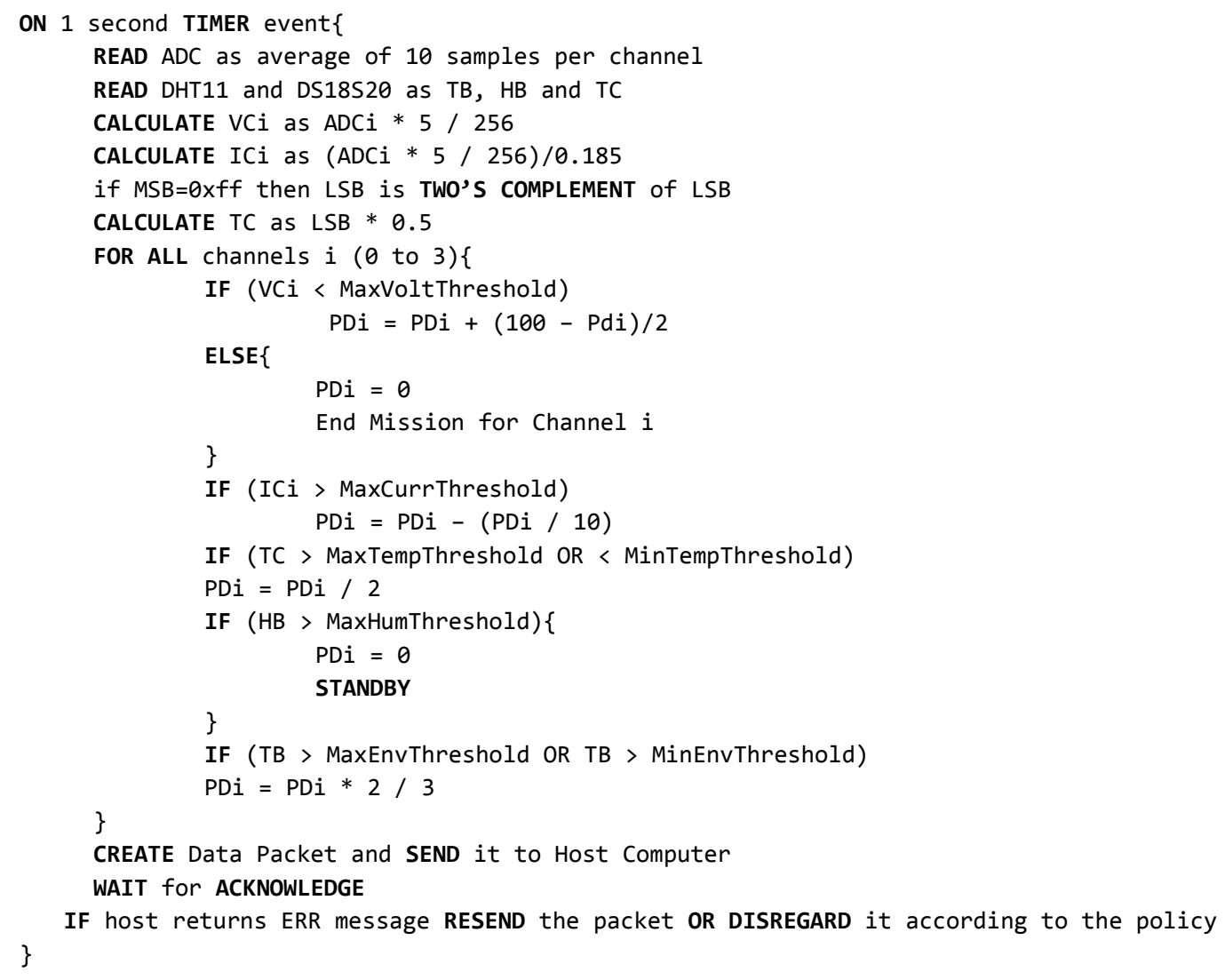

The algorithm executes the same event at each second. ADC values as well as sensors are read and corresponding variables take values. Each channel is controlled by a separate PWM signal. The charge pump starts logarithmically and feeds cells. If the charge current flow is more than preferred threshold it reduces the charge activity by 10 percent, per sampling iteration until it stabilizes the current flow. Cell temperature decreases the charge pump activity of corresponding cell logarithmically.

On the other hand, board temperature affects the PWM with a lower ratio than cell temperature. An extraordinary level of humidity protects the charging mechanism by changing its state to standby.

Data Packet is generated and is sent to the host computer waiting for the acknowledgement. If transmitted CRC would not match with the calculated one at the host controller, an ERR message is returned. This can be considered to repeat the previous message immediately or ignore it due to the nature of the application.

\subsection{Communication Protocol}

Captured temperature and humidity values from DHT11 sensor is used to protect the charging board against environmental failures. Therefore, TB and HB values are not transferred to the host computer. The remaining values are transferred in 12 bytes data packet format as follows: 


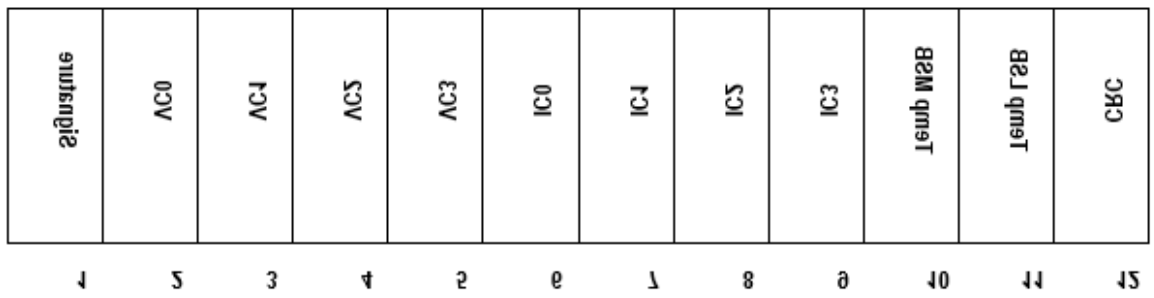

Figure.2 Transferred Data packet Structure

Signature byte is the fixed OXFF value as the start byte for data packet transfer. VCi and ICi values are 8bit resolution result of ADC module presenting $195 \mathrm{mv}$ per unit and $185 \mathrm{mv} / \mathrm{A}$. TC is transferred according to DS18S20 data register standard. It takes $750 \mathrm{~ms}$ at most to read the temperature value. The host emulator software reads the data packet and records processed information in a spreadsheet file.

In case of any OS interruption to the host program, it searches for the next received packet starting by packet signature. CRC is calculated at host to ensure the proper data transfer from MCU. An Acknowledge message is sent to MCU upon appropriate data transfer while an error message is sent in case of data corruption. Due to the real-time nature of the mission critical charging application, corrupted packet might not be retransferred to the host. Finally, MCU sends a packet with all capturing values as OXFF meaning the process termination.

Following figure presents the Adamus emulator while listing the available host serial ports to initiate data retrieval.

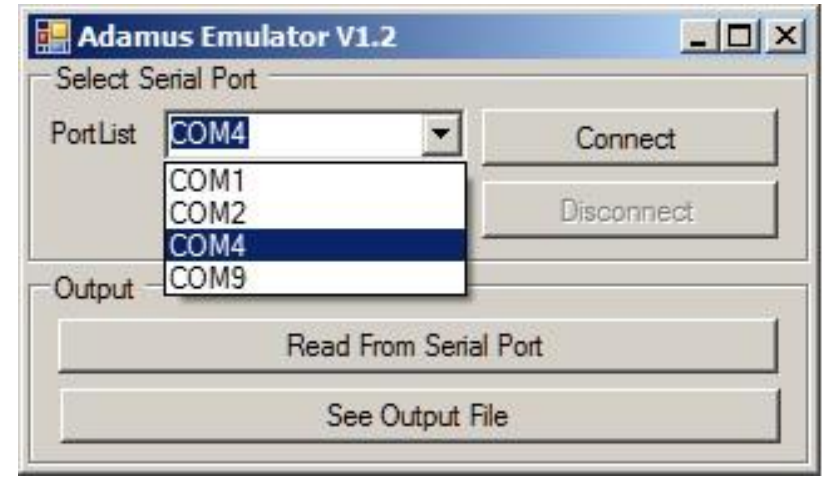

Figure 3. Adamus Emulator Software Interface

Other required commands can be easily defined and being transferred to the host device or reversely to the MCU by changing the initial signature and packet structure.

\section{Conclusion \& Future Work}

This research presents the design and implementation of an embedded ultra-fast parallel lithium charging cell with consideration of extreme cell and board temperature and humidity conditions. Switching charge pump varies using variable PWM values per each channel. The interface is easily connected to the host device for data logging purposes.

As the future work, we wish to examine various types of lithium cells including Li-MnO2, Li-SOCl2, Li-I2, $\mathrm{Li}-\mathrm{CUS}$, Li-FeS2, Li-NiCoO2, Li/Al-MnO2, and Li/Al-V2O5 in laboratory while simulating extreme high and 
Arif Sari, Behnam Rahnama \& Ersin Caglar; Ultra-fast Lithium cell charging architecture for mission critical applications, Transactions on Machine Learning and Artificial Intelligence, Volume 2 No 5 Oct (2014); pp: 10-16

low temperature and humidity conditions. Surrounding pressure might be also taken into account in case mission critical applications such as charging circuitry for satellites are being examined as a case study.

\section{ACKNOWLEDGMENTS}

Authors would like to thank the European University of Lefke for providing a wide range of research facilities at Robotics and Chemistry laboratories.

\section{REFERENCES}

[1]. S. Lee, Y. Cho, HK. Song, KT. Lee, J. Cho (2012). Angewandte Chemie, “Carbon-Coated Single-Crystal LiMn2O4 Nanoparticle Clusters as Cathode Material for High-Energy and High-Power Lithium-lon Batteries", Vol. 124, Issue 35, pp. 8878-8882, DOI: 10.1002/ange.201203581.

[2]. N. Li, Z. Chen, W. Ren, F. Li, H.-M. Cheng (2012). Proceedings of the National Academy of Sciences, "Flexible Graphene-based lithium ion batteries with ultrafast charge and discharge rates", Vol. 109, Issue 43, 17360.

[3]. HW. Lu, W. Zeng, YS. Li, ZW. Fu (2007). Journal of Power Sources, "Fabrication and Electrochemical properties of three-dimensional net architectures of anatase $\mathrm{TiO}_{2}$ and spinel Li4Ti5O12 nanofibers", Vol. 164, Issue 2, pp. 874-879,

[4]. DOI: 10.1016/j.jpowsour.2006.11.009

[5]. Z. Liang, HL. Pan, YS. Hu, L. Hong, C. Li-Quan (2012). China Physics B. "Spinel lithium titanate $\left(\mathrm{Li}_{4} \mathrm{Ti}_{5} \mathrm{O}_{12}\right)$ as a novel anode material for room-temperature sodium-ion battery", Vol. 12, No. 2, p. 028201-4. DOI: 10.1088/1674$1056 / 21 / 2 / 028201$.

[6]. L. Hu, H. Wu, F. La Manita, Y. Yang, Y. Cui, (2010). American Chemical Society Nano. "Thin, Flexible Secondary Li-ion Paper Batteries", Vol 26, No.4, p.5843-8, DOI: 10.1021/nn1018158.

[7]. HK. Liu, GX. Wang, Z. Guo, J. Wang, K. Konstantiov (2006). Journal of Nanoscience and Nanotechnology, "Nanomaterials for lithium-ion rechargeable batteries", Vol 6, No.1, pp. 1-15.

[8]. F. Cheng, J. Liang, Z. Tao, J. Chen (2011). Advanced Materials, "Functional Materials for rechargeable batteries", Vol 23., No.15, p.1695-715, DOI: 10.1002/adma.201003587 